\title{
Ultra-low-noise Regenerative Frequency Divider for High-Spectral-Purity RF Signal Generation
}

\author{
A. Hati, C. W. Nelson, C. Barnes, D. Lirette, J. A. DeSalvo, T. Fortier, F. Quinlan, A. Ludlow, \\ T. Rosenband, S. A. Diddams and D. A. Howe \\ Time and Frequency Division \\ National Institute of Standards and Technology \\ Boulder, CO 80305, USA \\ archita@boulder.nist.gov
}

\begin{abstract}
We implement an ultra-low-noise frequency divider chain from $8 \mathrm{GHz}$ to $5 \mathrm{MHz}$ that utilizes custom-built regenerative frequency divide-by-2 circuits. The single-sideband (SSB) residual phase-noise of this regenerative divider at $5 \mathrm{MHz}$ output is $-163 \mathrm{dBc} / \mathrm{Hz}$ at $10 \mathrm{~Hz}$ offset frequency. This level is achieved with custom-built mixers that use 2 N2222A bipolar junction transistors in a conventional double-balanced diode ring. We report absolute phase-noise of radio- frequency (RF) signals at $10 \mathrm{MHz}$ and $5 \mathrm{MHz}$ obtained by dividing an $8 \mathrm{GHz}$ signal generated from ultra-stable optical comb-based frequency division. The absolute SSB phase-noise for a $10 \mathrm{MHz}$ and $5 \mathrm{MHz}$ signal at $1 \mathrm{~Hz}$ offset is $-143 \mathrm{dBc} / \mathrm{Hz}$ and $-150 \mathrm{dBc} / \mathrm{Hz}$, and at $100 \mathrm{kHz}$ offset is $-174 \mathrm{dBc} / \mathrm{Hz}$ and $-177 \mathrm{dBc} / \mathrm{Hz}$, respectively.
\end{abstract}

\section{INTRODUCTION}

Frequency dividers are important building blocks used in a wide variety of microwave and radio-frequency (RF) system designs. One form of divider, the regenerative frequency divider, is very useful in low-phase-noise frequency synthesis [1-4]. These dividers can achieve lower residual phase-noise than other analog and digital dividers [5]. There are several emerging technologies that produce ultra-low phase-noise microwave and RF signals generated either from the optical comb-based division of a cavity-stabilized laser [6-8], or from a cryo-cooled sapphire microwave oscillator $[9,10]$. Frequency division by $\mathrm{N}$ reduces the signal phase-noise by $\mathrm{N}^{2}$. Therefore, by dividing an ultra-low-noise microwave signal that has SSB phase-noise, $\mathscr{L}(f)$, equal to $-104 \mathrm{dBc} / \mathrm{Hz}$ at 10 $\mathrm{GHz}$ [7], a very-low-noise RF signal can potentially be generated. An ideal division of this signal should produce -170 $\mathrm{dBc} / \mathrm{Hz}$ at $5 \mathrm{MHz}$. While this ideal phase-noise level may be below that of the best current technology, such ultra-low-noise levels could enable future applications in precision timing or navigation. Moreover, it is important to test and understand the absolute limits of optical and electronic frequency division.

In this paper, we begin by briefly describing a regenerative divide-by-2 circuit. This divider is designed with a custombuilt mixer $[11,12]$, and we achieved $\mathrm{L}(10 \mathrm{~Hz})$ equal to -163 $\mathrm{dBc} / \mathrm{Hz}$, when the divider operates at input frequencies of 40 $\mathrm{MHz}$ and lower.

We also describe the design and implementation of an ultra-low noise frequency synthesizer (or divider chain) for generation of low-noise RF signals. These signals are

Work of U. S. Government. Not Subject to Copyright. obtained by dividing an ultra-low phase-noise $8 \mathrm{GHz}$ microwave signal with a combination of digital and analog regenerative dividers. The $8 \mathrm{GHz}$ signal is generated via optical-comb frequency division [7].

\section{DESCRIPTION OF THE REGENERATIVE DIVIDER}

The basic block diagram of a regenerative divider is shown in Fig. 1. It consists of a mixer, amplifier, low-pass filter and phase shifter. A regenerative frequency divider multiplies the input signal $\left(f_{\text {in }}\right)$ with the feedback signal $\left(f_{\text {in }} / 2\right)$ from the mixer. This produces sum $\left(3 f_{\text {in }} / 2\right)$ and difference $\left(f_{\text {in }} / 2\right)$ frequencies at the output of the mixer. A low-pass filter (LPF) is used after the mixer to remove the undesired sum frequency, and the $f_{\text {in }} / 2$ frequency is amplified and fed back into the mixer. A second LPF is used after the loop amplifier to remove the thermal noise generated by the amplifier at $3 f_{\text {in }} / 2$ [3]. The phase-noise of the divider is given by $[3,4]$

$$
\mathscr{L}(f)_{\text {Div }}=\sum \mathscr{L}(f)_{\text {comp }} / N^{2},
$$

where $\mathscr{L}(f)_{\text {comp }}$ is the phase noise of the loop components.

Our design uses a custom-built mixer. It is a double-balanced mixer with four 2N2222A bipolar junction transistors in the diode ring. The collector and base of each transistor are tied together to form a diode ring, as shown in the mixer block of Fig. 1. The loop amplifier is a commercially available lownoise amplifier. The gain, noise figure and output power at the $1 \mathrm{~dB}$ compression point of the amplifier are $15 \mathrm{~dB}, 4 \mathrm{~dB}$ and $20 \mathrm{dBm}$, respectively. In a regenerative divider, either the mixer or the amplifier, or both, need to operate in compression. In our divider design, it is the mixer that limits the loop gain. The residual flicker phase-noise of the mixer and the amplifier chosen for the divider are roughly equal.

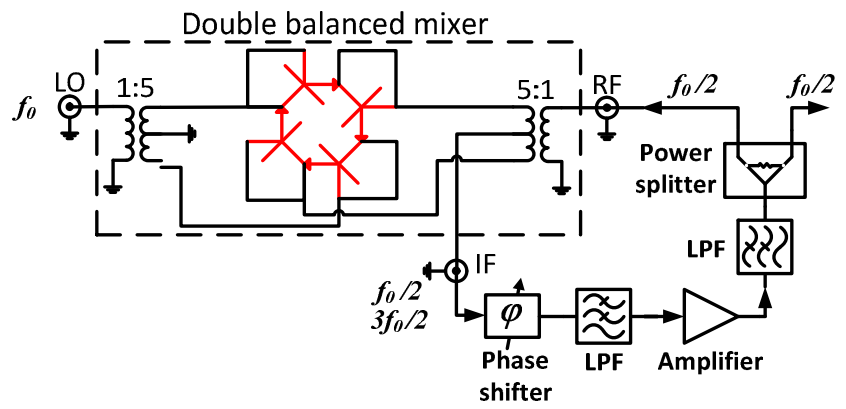

Figure 1. Block diagram of a regenerative divider. The diode ring in this double-balanced mixer is constructed by use of transistors with the collector input tied to the base. 


\section{RESidual PhaSe-NOISE AND Allan DEVIATION OF THE REGENERATIVE DIVIDER}

To start, we construct regenerative divide-by-2 circuits that operate at input frequencies of $20 \mathrm{MHz}$ and $10 \mathrm{MHz}$ and measure the residual phase-noise. In order to measure the noise of a single regenerative divider as a device under test (DUT), we build three similar dividers. A cross-spectrum measurement system [13] is designed with the same custombuilt mixers implemented as phase detectors. The experimental set-up is shown in Fig. 2, and the noise floor of the measurement system is shown in Fig. 3.

The loop phase and amplitude of the DUT are optimized for the lowest noise [2]. The residual output referred phasenoise of a single divider, $\mathrm{L}(10 \mathrm{~Hz})$, is approximately equal to $-163 \mathrm{dBc} / \mathrm{Hz}$, as shown in Fig. 3. Its $1 / f$ noise level is almost $10 \mathrm{~dB}$ better than any commercial regenerative divider available at these frequencies.

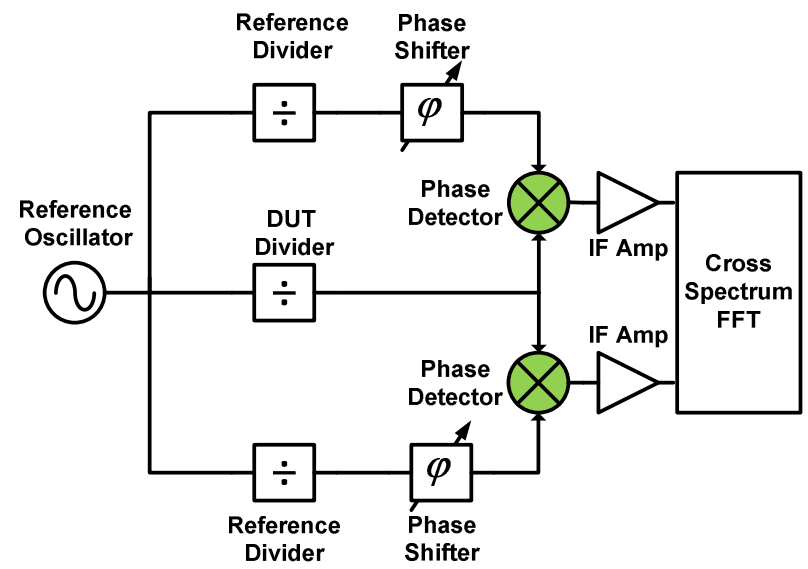

Figure 2. Experimental set-up for the divider phase noise measurement. IF AMP- Intermediate Frequency Amplifier, FFT - Fast Fourier Transform.

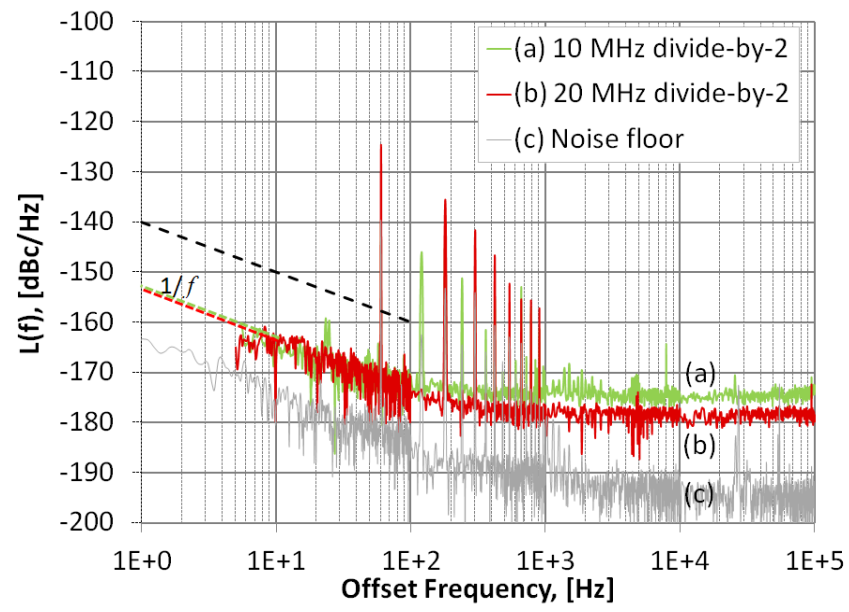

Figure 3. Single-sideband output referred phase-noise of the regenerative divider. (a) $10 \mathrm{MHz}$ divide-by-2 (b) $20 \mathrm{MHz}$ divide-by-2 (c) Noise floor. Dashed line is the typical noise of commercial regenerative dividers.

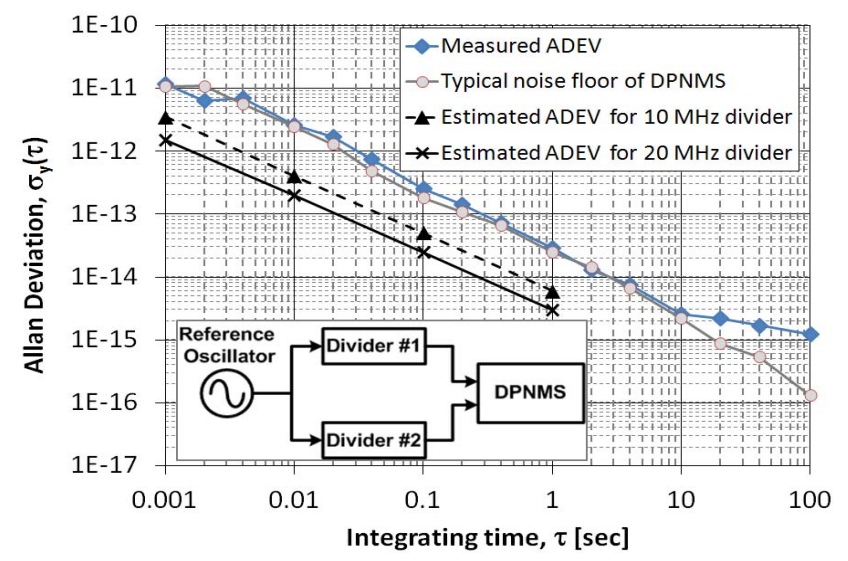

Figure 4. Residual Allan deviation (ADEV) of $10 \mathrm{MHz}$ and $20 \mathrm{MHz}$ divideby-2 circuits. The estimated ADEV is mapped from the measured phase noise. The inset shows the set-up for measuring $\sigma_{y}(\tau)$ of a pair of dividers. DPNMS - Digital Phase-Noise Measurement System.

To get an idea of the divider's performance in timing applications, the residual Allan deviation, $\sigma_{\mathrm{y}}(\tau)$ of a pair of dividers is measured at $10 \mathrm{MHz}$ and $5 \mathrm{MHz}$ with a digital phase-noise measurement system that computes the Allan deviation [14]. The measurement set-up is shown in the inset of Fig. 4. The measured $\sigma_{\mathrm{y}}(\tau)$ of both dividers is lower than the noise floor of the measurement system for an integration time $\tau<10 \mathrm{~s}$. For $1 \mathrm{~ms}<\tau<1 \mathrm{~s}, \sigma_{\mathrm{y}}(\tau)$ mapped from the residual phase-noise of Fig. 3 is shown. The $\sigma_{y}(\tau)$ at 0.1 sis estimated to be $5 \times 10^{-14}$ for a $10 \mathrm{MHz}$ divider and $3 \times$ $10^{-14}$ for a $20 \mathrm{MHz}$ divider, as shown in Fig. 4.

\section{Design AND IMPLementation Of AN UltRA-Low NOISE FREQUENCY SYNTHESIZER}

The block diagram of a frequency synthesizer based on optical frequency comb divider (OFCD) and a combination of digital and analog regenerative dividers is shown in Fig. 5. The input signal at $8 \mathrm{GHz}$ is generated from a cavitystabilized, self-referenced, $1 \mathrm{GHz}$ Ti:sapphire mode-locked laser [7]. The first two stages of the synthesizer are digital dividers and the remaining six dividers are regenerative. The custom-built mixers are used in the final three division stages. This synthesizer generates eight outputs, viz., $1.6 \mathrm{GHz}, 320$ $\mathrm{MHz}, 160 \mathrm{MHz}, 80 \mathrm{MHz}, 40 \mathrm{MHz}, 20 \mathrm{MHz}, 10 \mathrm{MHz}$ and 5 $\mathrm{MHz}$ when an $8 \mathrm{GHz}$ signal is applied at the input. Several other frequencies can be generated by mixing two signals; for example, a $100 \mathrm{MHz}$ signal can be obtained by mixing 80 $\mathrm{MHz}$ and $20 \mathrm{MHz}$. The synthesizer operates at input powers anywhere between $-5 \mathrm{dBm}$ to $+10 \mathrm{dBm}$ and the output power level of all stages is approximately $11 \mathrm{dBm}$. An advantage of having a digital divider at the input is that it reduces the amplitude-modulated (AM) noise of the $8 \mathrm{GHz}$ signal.

We built two identical divider chains similar to that shown in Fig. 5 and measured the residual phase-noise of the entire chain at $10 \mathrm{MHz}$ and $5 \mathrm{MHz}$ outputs. The results are shown in Fig. 6 in dotted lines. The SSB residual noise at $1 \mathrm{~Hz}$ offset is $-150 \mathrm{dBc} / \mathrm{Hz}$ and $-146 \mathrm{dBc} / \mathrm{Hz}$. 


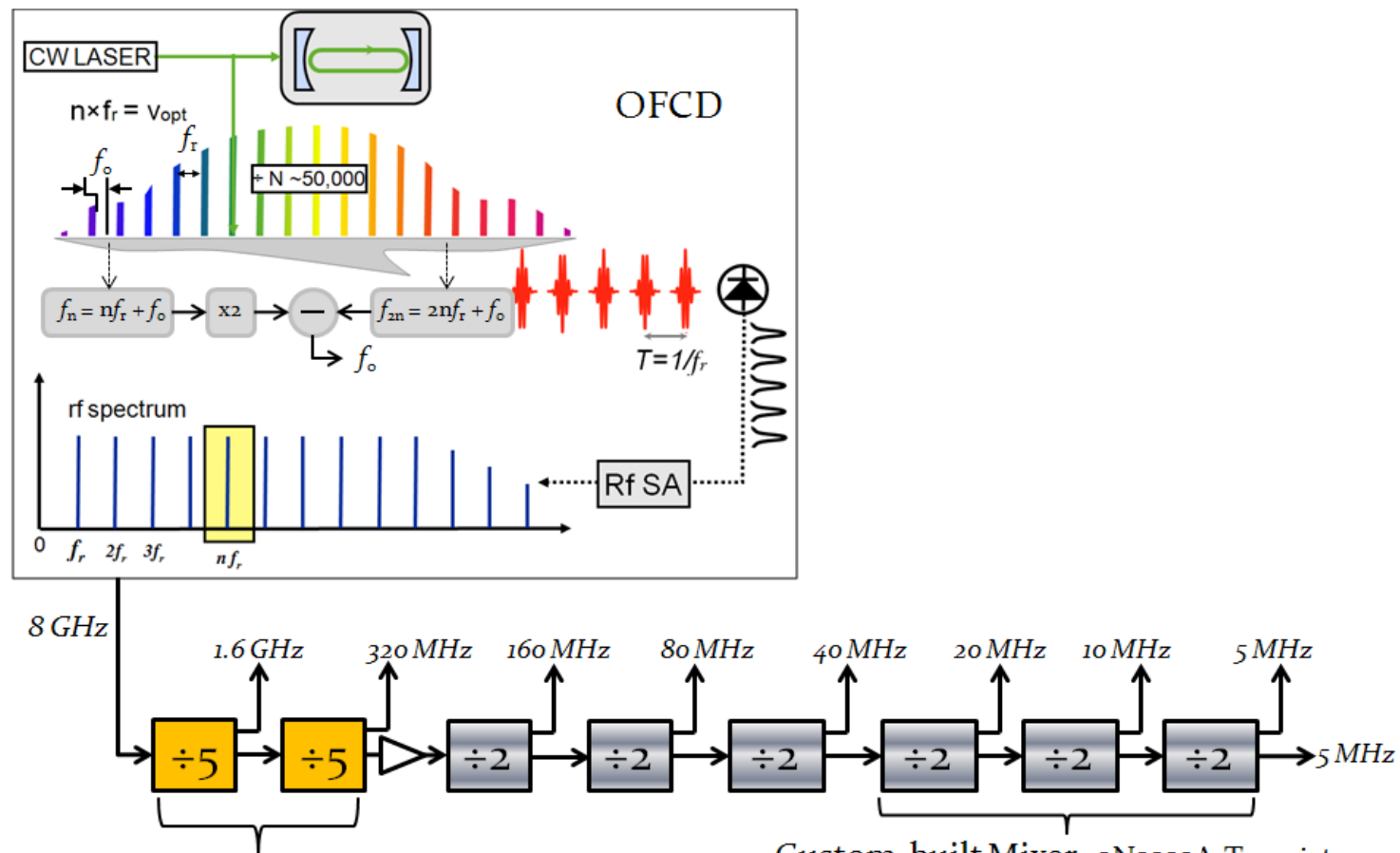

Digital Dividers

Custom-built Mixer-2N2222A Transistors

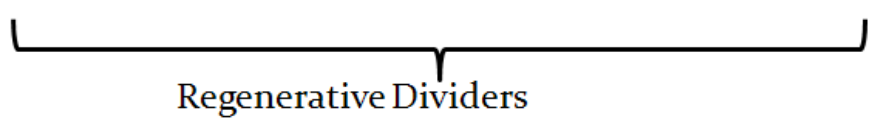

Figure 5. Block diagram of the frequency synthesizer. OFCD - Optical frequency comb divider.

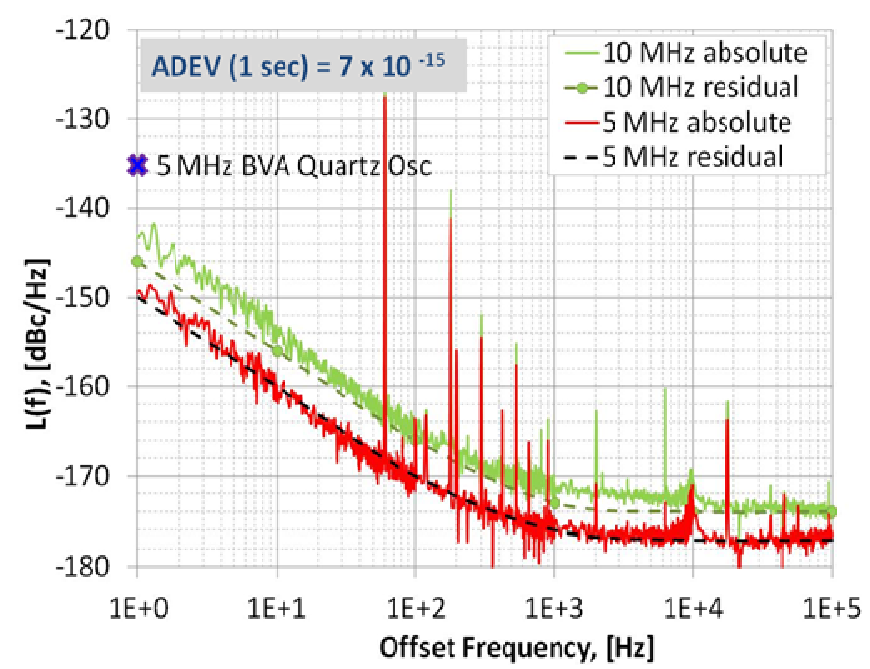

Figure 6. Absolute phase-noise of the synthesized signals at $5 \mathrm{MHz}$ and 10 MHz. The dashed lines represent the residual phase-noise of the divider chain.

In addition to residual noise, the absolute phase-noise of the synthesized $5 \mathrm{MHz}$ and $10 \mathrm{MHz}$ signals is also measured. The $8 \mathrm{GHz}$ signals for each divider chain are generated from two similar but independent OFCDs, each phase-locked to its own independent cavity-stabilized lasers $[15,16]$. The results of the absolute noise are shown in Fig. 6. We achieve a SSB absolute phase-noise of $\mathrm{L}(1 \mathrm{~Hz})=-143 \mathrm{dBc} / \mathrm{Hz}$ and -150 $\mathrm{dBc} / \mathrm{Hz}$ and $\mathrm{L}(100 \mathrm{kHz})=-174 \mathrm{dBc} / \mathrm{Hz}$ and $-177 \mathrm{dBc} / \mathrm{Hz}$ for
$10 \mathrm{MHz}$ and $5 \mathrm{MHz}$ signals, respectively. This corresponds to a frequency stability of $7 \times 10^{-15}$ at $1 \mathrm{~s}$ averaging time. The noise of the $5 \mathrm{MHz}$ signal coincides with and is limited by the divider noise. However, in the $10 \mathrm{MHz}$ case, the absolute noise close-to carrier is a few decibels higher that the residual noise indicated by the dashed line, due to unmitigated groundloop effects. The close-to-carrier phase-noise and short-term instability at these frequencies generated from our frequency synthesizer are lower than that achieved with any other synthesizer or signal source. For comparison, the state-of-theart high stability $\mathrm{BVA}^{1}$ quartz oscillator at $5 \mathrm{MHz}$ has phasenoise, $\mathrm{L}(1 \mathrm{~Hz})=-135 \mathrm{dBc} / \mathrm{Hz}$ [17], almost $15 \mathrm{~dB}$ higher than the signal generated from optical division.

\section{CONCLUSION}

We report an ultra-low-noise regenerative frequency divider. The low noise of the divider is achieved by use of custom-built mixers with 2N2222A bipolar junction transistors (BJTs) in a conventional double-balanced diode ring. We also report absolute phase-noise of RF signals at 10 $\mathrm{MHz}$ and $5 \mathrm{MHz}$ obtained by dividing an $8 \mathrm{GHz}$ signal generated from ultra-stable optical-comb-based frequency division. The SSB absolute phase-noise achieved for $10 \mathrm{MHz}$ and $5 \mathrm{MHz}$ signals is $\mathrm{L}(1 \mathrm{~Hz})=-143 \mathrm{dBc} / \mathrm{Hz}$ and $-150 \mathrm{dBc} / \mathrm{Hz}$ and $\mathrm{L}(100 \mathrm{kHz})=-174 \mathrm{dBc} / \mathrm{Hz}$ and $-177 \mathrm{dBc} / \mathrm{Hz}$, respectively. The close-to-carrier phase-noise and short-term

\footnotetext{
${ }^{1}$ Manufacturer is indicated for information. No endorsement is implied.
} 
instability at these frequencies generated from our frequency synthesizer are lower than that achieved with any other synthesizer or signal source.

In the future, we would like to optimize the noise of each stage of the divider chain, package the entire divider chain in a rack-mount enclosure for improved long-term stability and environmental isolation.

\section{REFERENCES}

[1] R. L. Miller, "Fractional-frequency generators utilizing regenerative modulation," Proc. Inst. Radio Eng., vol. 27, pp. 446- 4572, 1939.

[2] E. Rubiola, M. Olivier, J. Groslambert, "Phase-noise in the regenerative frequency dividers," IEEE Trans Instrum. Meas., vol. 41, no. 3, p. 353360, June 1992.

[3] E.S. Ferre-Pikal and F.L. Walls, "Microwave Regenerative Dividers with Low Phase-noise," IEEE Trans. Ultrason. Ferroelectr. Freq. Control, vol. 46, pp. 216-219, 1999.

[4] A. Sen Gupta, J.F. Garcia Nava, and F.L. Walls, "A novel low noise regenerative divide-by-four circuit," in Proc. IEEE 2002 Intl. Freq. Cont. Symp., pp. 680-684, 2002.

[5] M. M. Driscoll and T. D. Merrell, "Spectral performance of frequency multipliers and dividers," in Proc. IEEE Freq. Contr. Symp., pp. 193200, 1992.

[6] S. A. Diddams, A. Bartels, T. M. Ramond, C. W. Oates, S. Bize, E. A. Curtis, J. C. Bergquist, and L. Hollberg, "Design and control of femtosecond lasers for optical clocks and the synthesis of low noise optical and microwave signals," IEEE Journ. Select. Topics Quant. Electron. vol. 9, pp. 1072, 2003.

[7] T.M. Fortier, M.S. Kirchner, F. Quinlan, J. Taylor, J.C. Bergquist, T. Rosenband, N. Lemke, A. Ludlow, Y. Jiang, C.W. Oates, and S.A.
Diddams, "Generation of ultrastable microwaves via optical frequency division," Nature Photonics, vol. 5, pp. 425-429, 2011.

[8] W.C. Swann, E. Baumann, F. R. Giorgetta and N. Newbury, "Microwave generation with low residual phase-noise from a femtosecond fiber laser with an intracavity electro-optic modulator," Optics Express, vol. 19, no. 24, pp. pp. 24387-24395, Nov. 2011.

[9] S. Grop, P.Y. Bourgeois, E. Rubiola, W. Schäafer, J. De Vicente, Y. Kersale, V. Giordano, "Frequency synthesis chain for the ESA Deep Space Network," Electron. Lett., vol. 47, no. 6, pp. 386-388, 2011.

[10] N. R. Nand, J. G. Hartnett, E. N. Ivanov, and G. Santarelli, "Ultrastable very-low phase-noise signal source for very long baseline interferometry using a cryocooled sapphire oscillator," IEEE Trans Microw. Theory Tech., vol. 59, no. 11, pp. 2978- 2986, Nov. 2011.

[11] C. Barnes, A. Hati, C.W. Nelson, and D.A. Howe, "Residual PM Noise Evaluation of Radio Frequency Mixers," in Proc. 2011 Joint Mtg. IEEE Intl. Freq. Cont. Symp. and EFTF Conf., pp. 724-728, 2011.

[12] C. Barnes, A. Hati, C.W. Nelson, and D.A. Howe, "5 MHz Phase Detector With Low Residual Flicker," Electron. Lett., vol. 47, no. 19, pp. 1066-1067, 2011.

[13] K. H. Sann, "The measurement of near-carrier noise in microwave amplifiers," IEEE Trans. Microw. Theory Tech., pp. 761-766, 1968.

[14] J. Grove, J. Hein, J. Retta, P. Schweiger, W. Solbrig, and S. R. Stein, "Direct-digital phase-noise measurement," in Proc. IEEE Freq. Contr. Symp., pp. 287-291,2004.

[15] A. Ludlow, Y. Jiang, N. Lemke, R. Fox, J. Sherman, L. Ma, and C. Oates, "Making optical atomic clocks more stable with $10^{\wedge}-16$ level laser stabilization," Nature Photonics, vol. 5, pp. 158-161, 2011.

[16] B. C. Young, F.C. Cruz, J.C. Bergquist, and W.M. Itano, "Visible Lasers with Subhertz Linewidths," Phys. Rev. Lett., vol. 82, pp. 3799$3802,1999$.

[17] J. Chauvin, P. Weber, J. P, Aubry, F. Lefebvre, F. Sthal, S. Galliou, E. Rubiola, X. Vacheret, "A New Generation of Very High Stability BVA Oscillators," in Proc. 2007 Joint Mtg. IEEE Intl. Freq. Cont. Symp. and EFTF Conf., pp. 1261-1268, 2007. 